\title{
Mechanical feedback in the molecular ISM of luminous IR galaxies
}

\author{
A. F. Loenen ${ }^{1,2}$, M. Spaans ${ }^{1}$, W. A. Baan ${ }^{2}$, and R. Meijerink ${ }^{3}$ \\ 1 Kapteyn Astronomical Institute, PO Box 800, 9700 AV Groningen, The Netherlands \\ e-mail: loenen@astro.rug.nl \\ 2 ASTRON, PO Box 2, 7990 AA Dwingeloo, The Netherlands \\ 3 Astronomy Department, University of California, Berkeley, CA 94720, USA \\ Received 4 June 2008 / Accepted 4 July 2008
}

ABSTRACT

\begin{abstract}
Aims. Molecular emission lines originating in the nuclei of luminous infra-red galaxies are used to determine the physical properties of the nuclear ISM in these systems.

Methods. A large observational database of molecular emission lines is compared with model predictions that include heating by UV and X-ray radiation, mechanical heating, and the effects of cosmic rays.

Results. The observed line ratios and model predictions imply a separation of the observed systems into three groups: XDRs, UV-dominated high-density $\left(n \geq 10^{5} \mathrm{~cm}^{-3}\right)$ PDRs, and lower-density $\left(n=10^{4.5} \mathrm{~cm}^{-3}\right)$ PDRs that are dominated by mechanical feedback.

Conclusions. The division of the two types of PDRs follows naturally from the evolution of the star formation cycle of these sources, which evolves from deeply embedded young stars, resulting in high-density $\left(n \geq 10^{5} \mathrm{~cm}^{-3}\right)$ PDRs, to a stage where the gas density has decreased $\left(n=10^{4.5} \mathrm{~cm}^{-3}\right)$ and mechanical feedback from supernova shocks dominates the heating budget.
\end{abstract}

Key words. ISM: molecules - galaxies: ISM - galaxies: starburst - galaxies: active - infrared: galaxies - radio lines: galaxies

\section{Introduction}

Dense $\left(n>10^{4} \mathrm{~cm}^{-3}\right.$ ) molecular gas plays an important role in the physics of (Ultra-) Luminous Infrared Galaxies. It provides the materials from which stars are formed and fuels possible active galactic nuclei. The radiation originating in molecular gas in the nuclei of these galaxies provides information about the physical properties of the nuclear environment, such as the gas density and chemical composition, and the dominant type of radiation field. There are two important types of radiation: UV radiation $(6-13.6 \mathrm{eV})$ in star-forming regions, which generates Photon Dominated Regions (PDRs; e.g. Hollenbach \& Tielens 1999); and X-rays (1-100 keV) emanating from accreting black holes, which produce in X-ray Dominated Regions (XDRs; e.g. Lepp \& Dalgarno 1996; Maloney et al. 1996). Analysis of the state of molecular gas in a significant number of galaxies allows systematic effects to be identified, which can provide insight into the processes influencing the (gas in the) nuclei of these galaxies: the star-formation rate, the evolutionary stage of the system, and feedback processes.

Baan et al. (2008) presented data for the CO, HCN, HNC, $\mathrm{HCO}^{+}, \mathrm{CN}$, and $\mathrm{CS}$ line emission of 37 infrared luminous galaxies and 80 additional sources taken from the literature (e.g., Nguyen-Q-Rieu et al. 1992; Solomon et al. 1992; Aalto et al. 2002; Gao \& Solomon 2004; Graciá-Carpio et al. 2006). They suggested that the sources can be divided into PDRs and XDRs, based on the ratios of emission lines. We expand on their analysis and explore in more detail the different physical and chemical processes responsible for the diversity in line ratios found in the Baan et al. (2008) data, by comparing their data with the predictions of PDR and XDR chemistry models first presented by Meijerink \& Spaans (2005).

\section{PDR and XDR models}

Figure 1 shows the $\mathrm{HCN}, \mathrm{HNC}$ and $\mathrm{HCO}^{+}$line ratios from Baan et al. (2008). To identify the physical processes that generate the line ratios measured, we compare the data with the predictions of a large number of PDR and XDR models. Meijerink \& Spaans (2005) and Meijerink et al. (2007) presented a code to model one-dimensional PDR and XDR clouds. It takes into account the various heating and cooling processes as well as the chemistry of the gas. Because the excitation of the molecular species is included, the intensities of individual molecular emission lines can be calculated as well. Our model predictions for both PDRs and XDRs are shown in Fig. 1 by solid lines: the green and cyan lines show the XDR results and the blue, purple, and red ones the PDRs. These lines represent the line ratios emanating from different depths in the cloud, and cover a column density range from about $N_{\mathrm{H}}=10^{21.5} \mathrm{~cm}^{-2}$ up to $10^{22}-10^{24} \mathrm{~cm}^{-2}$ (depending on the density of the cloud). Three conclusions can be drawn from this comparison:

1: PDRs and XDRs can be separated using the HNC/HCN line ratio. In XDRs, HNC is always stronger than the HCN line, producing a line ratio higher than unity, whereas for PDRs the inverse is generally true (see Figs. 1b,c);

2: in the case of PDR chemistry, models with different densities can be distinguished using the ratio of $\mathrm{HCO}^{+}$to $\mathrm{HCN}$ or HNC, which decreases with increasing density (see Fig. 1a). This follows from the increased dissociative recombination rate of $\mathrm{HCO}^{+}$with free electrons. A change in UV flux of two orders of magnitude produces only modest changes in the line ratios, because the UV field is significantly attenuated at the high column densities where the molecules are abundant; 


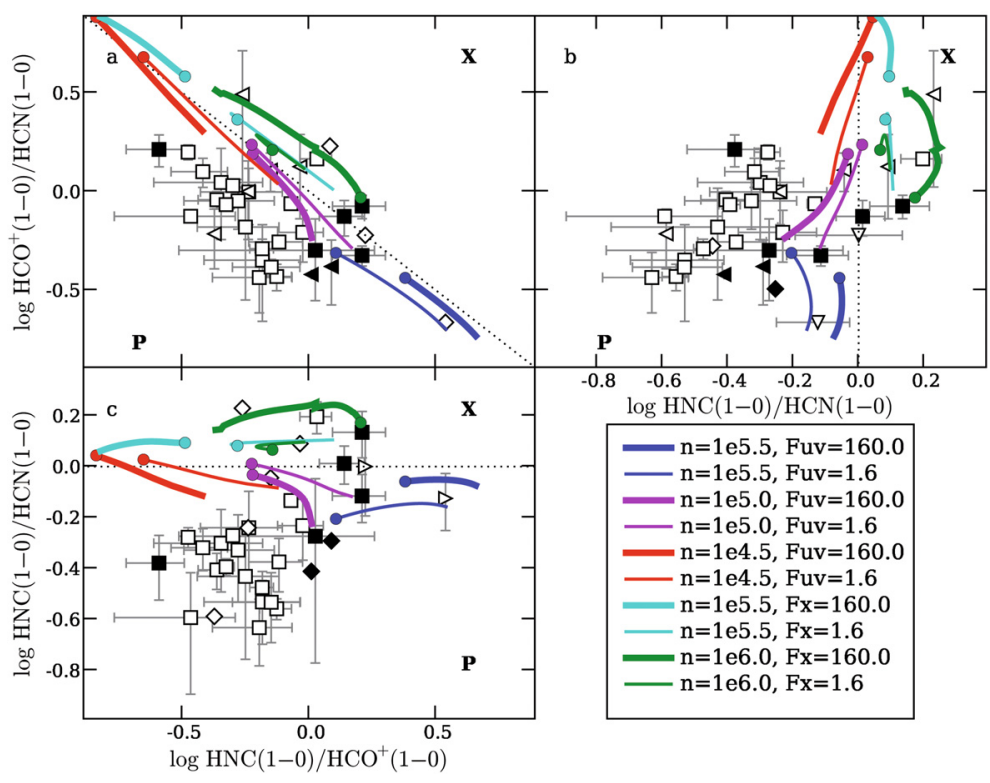

Fig. 1. Diagnostic diagrams using integrated line ratios of $\mathrm{HCN}$, $\mathrm{HNC}$, and $\mathrm{HCO}^{+}$versus each other. a) Integrated $\mathrm{HCO}^{+} / \mathrm{HCN}$ versus $\mathrm{HNC} / \mathrm{HCO}^{+}$ratios, b) integrated $\mathrm{HCO}^{+} / \mathrm{HCN}$ versus $\mathrm{HNC} / \mathrm{HCN}$ ratios, and c) integrated $\mathrm{HNC} / \mathrm{HCN}$ versus $\mathrm{HNC} / \mathrm{HCO}^{+}$ratios. The symbols $\mathrm{X}$ and $\mathrm{P}$ and the dotted lines mark the regions of the line ratios of XDRs and PDRs. Squares indicate reliable values and triangles upper and lower limits. In addition, filled symbols indicate $\mathrm{OH}$ or $\mathrm{H}_{2} \mathrm{CO}$ megamasers. Colored lines indicate results for PDR and XDR modeling, as function of column density (see Sect. 2), where the dot denotes the highest column. See Table 1 and legend for details about the models; $n$ denotes the density in $\mathrm{cm}^{-3}$, and $F_{\mathrm{UV}}$ and $F_{\mathrm{X}}$, respectively, the UV and X-ray flux in $\mathrm{erg} \mathrm{s}^{-1} \mathrm{~cm}^{-2}$.
3: the most significant result from this comparison is that a significant part of the data points has $\mathrm{HNC} / \mathrm{HCN}$ and $\mathrm{HNC} / \mathrm{HCO}^{+}$line ratios that are systematically lower than those of the data points traced by the models $\left(\log (\mathrm{HNC} / \mathrm{HCN})<-0.2\right.$ and $\left.\log \left(\mathrm{HNC} / \mathrm{HCO}^{+}\right)<-0.1\right)$. A lower $\mathrm{HNC} / \mathrm{HCO}^{+}$ratio can be obtained by decreasing the density of the gas to $10^{4.5} \mathrm{~cm}^{-3}$ (red lines in Fig. 1), but the $\mathrm{HNC} / \mathrm{HCN}$ ratios remain lower than can be explained by the PDR and XDR models. Meijerink et al. (2006) investigated the effects of the extra heating and ionization due to Cosmic Rays (CRs) and showed that although CRs have an influence on $\mathrm{HCO}^{+}$, which is sensitive to the ionization balance of the gas, they have little influence on the abundances of $\mathrm{HCN}$ and HNC. All this indicates that the state of the gas is influenced by processes that were not incorporated into the Meijerink et al. (2007) models.

\section{Mechanical heating}

The observed low HNC/HCN ratios could be produced by an enhanced conversion of $\mathrm{HNC}$ into $\mathrm{HCN}$ in warm gas. At temperatures higher than $100 \mathrm{~K}, \mathrm{HNC}$ is converted efficiently into $\mathrm{HCN}$ $(\mathrm{HNC}+\mathrm{H} \rightarrow \mathrm{HCN}+\mathrm{H}$; Schilke et al. 1992; Talbi et al. 1996). Dense molecular gas temperatures in excess of $100 \mathrm{~K}$ have been observed. Ott et al. (2005) presented interferometric maps of $\mathrm{NH}_{3}$ of the central $500 \mathrm{pc}$ of the nearby starburst galaxy NGC 253 and found clumps with kinetic temperatures of between $\sim 150 \mathrm{~K}$ and $\sim 240 \mathrm{~K}$. A similar study of $\mathrm{H}_{2} \mathrm{CO}$ in the starburst galaxy M 82 by Mühle et al. (2007) found kinetic temperatures of between $\sim 160 \mathrm{~K}$ and $\sim 260 \mathrm{~K}$ and densities on the order of $10^{4} \mathrm{~cm}^{-3}$. We note that such temperatures will occur only in the dense gas in the centers of the galaxies in our sample. The large-scale low-density $\left(n<10^{4} \mathrm{~cm}^{-3}\right)$ molecular gas (as traced by $\mathrm{CO}$ ) has lower temperatures. In the PDR, XDR, and CR models, temperatures of $100 \mathrm{~K}$ or higher are only reached at the edge of the clouds, where the abundances of HCN and HNC are too low to be detectable. The regions deeper inside the clouds, where the molecules become abundant $\left(\right.$ at $N_{\mathrm{H}} \geq 10^{21.5} \mathrm{~cm}^{-2}$ ), are cooler than $100 \mathrm{~K}$ for radiative heating (see Fig. 3).

Since all radiative heating processes are insufficient to heat gas at larger depths into the cloud, we add mechanical heating to the models. Sources of this type of heating are discussed in the
Table 1. The important parameters for the PDR and XDR simulations and the line styles and widths used to plot the results in Figs. 1 and 2.

\begin{tabular}{lllllll}
\hline \hline & $\begin{array}{l}n \\
{\left[\mathrm{~cm}^{-3}\right]}\end{array}$ & $\begin{array}{l}F \\
{\left[\mathrm{erg} \mathrm{s}^{-1} \mathrm{~cm}^{-2}\right]}\end{array}$ & \multicolumn{2}{l}{$\Gamma_{\text {mech }}$} \\
{$\left[\mathrm{erg} \mathrm{s}^{-1} \mathrm{~cm}^{-3}\right]$} & \\
& & \multicolumn{4}{l}{$\begin{array}{l}\text { line width } \\
\text { thin thick }\end{array}$} & \multicolumn{2}{l}{$\begin{array}{l}\text { line style } \\
\text { solid }\end{array}$} & $\begin{array}{l}\text { dashed } \\
\text { Fig. 1 }\end{array}$ & Fig. 2 & Fotted \\
& & & & Fig. 2 \\
\hline PDR & $10^{4.5}$ & 1.6 & 160 & 0 & $3 \times 10^{-19}$ & $5 \times 10^{-19}$ \\
& $10^{5.0}$ & 1.6 & 160 & 0 & $2 \times 10^{-18}$ & $9 \times 10^{-18}$ \\
& $10^{5.5}$ & 1.6 & 160 & 0 & $9 \times 10^{-18}$ & $2 \times 10^{-17}$ \\
\hline XDR & $10^{5.5}$ & 1.6 & 160 & 0 & - & - \\
& $10^{6.0}$ & 1.6 & 160 & 0 & - & - \\
\hline
\end{tabular}

following section. The mechanical heating is implemented by adding a depth independent heating rate $\left(\Gamma_{\text {mech }}\right)$ to the total heating budget. A new grid of models was calculated for several densities, radiation fields, and mechanical heating rates (see Table 1) and the results were plotted in Fig. 2. The adopted mechanical heating rates were chosen in such a way that temperatures deep inside the cloud $\left(N_{\mathrm{H}} \geq 10^{21.5} \mathrm{~cm}^{-2}\right)$ were between $150 \mathrm{~K}$ and $250 \mathrm{~K}$. The mechanical heating rates are typically about 10-50 times lower than the radiative heating rate at the edge of the cloud, but start to dominate the heating balance deeper inside the cloud $\left(N_{\mathrm{H}} \geq 10^{21.5} \mathrm{~cm}^{-2}\right)$, where they are several orders of magnitude higher than both the radiative and $\mathrm{CR}$ heating rates. Figure 3 illustrates how the temperature increases when a mechanical heating term is added. It also demonstrates that the increase in temperature has the desired effect on the abundances of the species: even though the total abundances of both molecules in the cloud are enhanced, the abundance of $\mathrm{HCN}$ increases with respect to HNC by a factor of about three.

The resulting line ratios are plotted in Fig. 2 and show that, due to a decrease in the $\mathrm{HNC} / \mathrm{HCN}$ ratio, data that could not be explained previously are now described well by models with a density of $10^{4.5} \mathrm{~cm}^{-3}$ and a range of UV fluxes and mechanical heating rates (red lines). Although models with higher densities predict line strengths that are higher then the line strengths for the $n=10^{4.5} \mathrm{~cm}^{-3}$ models and should be detectable, they produce line ratios that are not observed (and therefore fall 


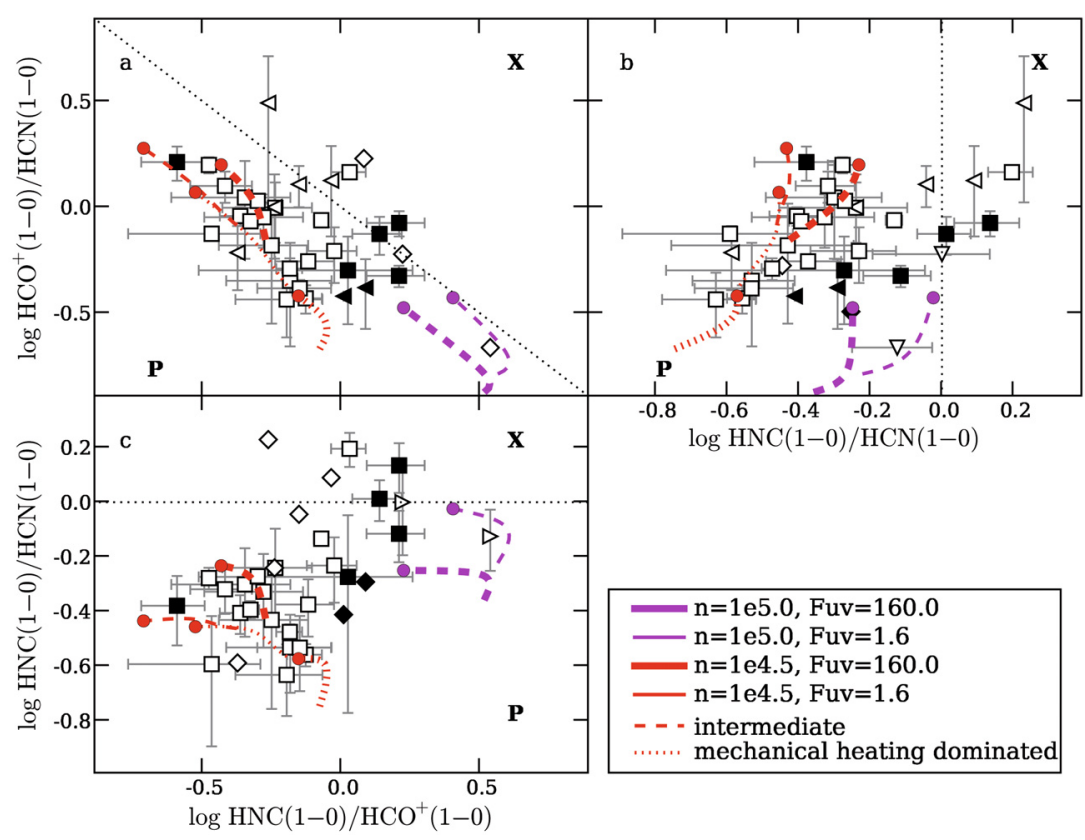

Fig. 2. Diagnostic diagrams that show the effects of the added mechanical heating. See the legend and Table 1 for details on the individual models. Plot symbols are similar to Fig. 1.

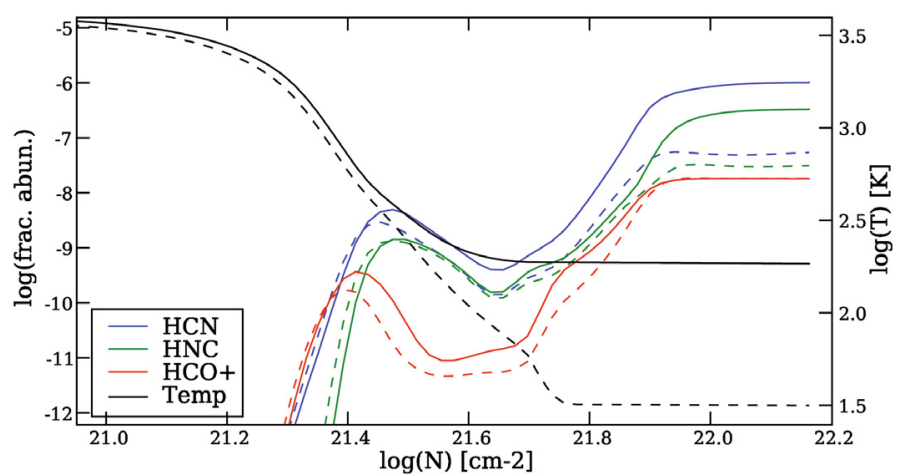

Fig. 3. Effects of mechanical heating on the temperature of the gas and the abundances of $\mathrm{HCN}, \mathrm{HNC}$, and $\mathrm{HCO}^{+}\left(n=10^{4.5} \mathrm{~cm}^{-3}, F_{\mathrm{UV}}=\right.$ $\left.160 \mathrm{erg} \mathrm{s}^{-1} \mathrm{~cm}^{-2}, \Gamma_{\text {mech }}=3 \times 10^{-19} \mathrm{erg} \mathrm{s}^{-1} \mathrm{~cm}^{-3}\right)$. Solid lines show the results of a model with mechanical heating, dashed lines for a model without.

outside the range of data points plotted in Fig. 2). An explanation for why these high-density systems are not observed is that the mechanical energies needed to heat the gas are unavailable (see Sect. 4).

Kohno et al. (2008) reported strong HCN emission in the cores of some Seyfert galaxies ("HCN-enhanced nuclei", or HENs). Because of the lack of evidence for a strong nuclear starburst (e.g. lack of mid-IR PAH emission), they attributed this to XDR chemistry. However, our results show that a weak PDR with mechanical heating can exhibit the same characteristics.

\section{Sources of mechanical heating}

The addition of mechanical heating to PDR clouds provides a satisfactory means of explaining the observations. However, we need to identify an available and sufficient source of energy. During the evolution of a starburst, there are two feedback processes that potentially provide sufficient mechanical energy to heat dense molecular gas to the required temperatures. In the early stage of nuclear star-formation (the first $10^{5}-10^{6}$ years), YSO outflows inject mechanical energy into individual clouds. Then, after about $10 \mathrm{Myr}$, the first massive $\left(M_{\star}>8 M_{\odot}\right)$ stars become supernovae ( $\mathrm{SNe})$, and inject mechanical energy into the star-forming region, which is distributed subsequently amongst the PDR clouds by turbulent dissipation. Since the energy injection required per cloud is known from the chemical modeling $\left(\Gamma_{\text {mech }}\right)$, the required YSO outflow velocities and $\mathrm{SN}$ rates for the different models can be calculated.

To estimate the effect of YSO outflows on the medium, we follow the recipe of Heckman et al. (1990) to determine the energy injection rate needed to create an YSO outflow bubble as:

$$
\frac{\mathrm{d} E_{\text {flow }}}{\mathrm{d} t} \approx 3 \times 10^{29} r_{\mathrm{pc}}^{2} v_{\text {flow }}^{3} n \mathrm{erg} \mathrm{s}^{-1},
$$

where $r_{\mathrm{pc}}$ is the size of the bubble in pc, $v_{\text {flow }}$ is the outflow velocity in $\mathrm{km} \mathrm{s}^{-1}$, and $n$ is the initial density of the medium in $\mathrm{cm}^{-3}$. By assuming that the outflow energy is distributed homogeneously over a cloud, of the same size as the bubble, the heating rate is the energy injection rate divided by the volume of the cloud: $\Gamma_{\text {mech }}=\frac{\mathrm{d} E_{\text {flow }}}{\mathrm{d} t} / V_{\text {cloud }}$. From this, the outflow velocity needed to produce a certain $\Gamma_{\text {mech }}$ in a cloud can be calculated (using a typical cloud size of $0.1 \mathrm{pc}$, which corresponds to a column density of $N_{\mathrm{H}} \approx 10^{22.2} \mathrm{~cm}^{-2}$ and a extinction of $A_{\mathrm{V}} \approx 15 \mathrm{mag}$ ) to be

$v_{\text {flow }} \approx\left(4.1 \times 10^{25} \Gamma_{\text {mech }} n^{-1}\right)^{\frac{1}{3}} \mathrm{~km} \mathrm{~s}^{-1}$.

This yields outflow velocities of around $10 \mathrm{~km} \mathrm{~s}^{-1}$ (see Table 2), which can be achieved in YSO outflows. However, this requires that every dense cloud has a YSO nearby, which is unlikely since the YSO phase is short-lived.

To determine the $\mathrm{SN}$ rate required to heat clouds on a global scale rather than for each individual cloud, we need to consider the size of the total starburst region (a sphere of diameter $D_{\mathrm{SB}}$ ), and the number of PDRs per $\mathrm{kpc}^{3}\left(f_{\mathrm{PDR}}\right)$. The transfer efficiency $\left(\eta_{\text {trans }}\right)$ parameterizes the dissipation of mechanical energy as heat as it cascades from the large scales of the SNe shock bubbles to the individual clouds. The total energy injection rate needed for the entire starburst region is then:

$\frac{\mathrm{d} E_{\mathrm{SN}}}{\mathrm{d} t}=\frac{\pi}{6} D_{\mathrm{SB}}^{3} f_{\mathrm{PDR}} V_{\text {cloud }} \Gamma_{\text {mech }} \operatorname{erg~s}^{-1}$ 
Table 2. A summary of the results of the calculations of the energy requirements of the models, as described in Sect. 4.

\begin{tabular}{lllll}
\hline \hline $\begin{array}{l}n \\
{\left[\mathrm{~cm}^{-3}\right]}\end{array}$ & $\begin{array}{l}\Gamma_{\text {mech }} \\
{\left[\mathrm{erg} \mathrm{s}^{-1} \mathrm{~cm}^{-3}\right]}\end{array}$ & $\begin{array}{l}v_{\text {flow }} \\
{\left[\mathrm{km} \mathrm{s}^{-1}\right]}\end{array}$ & $\begin{array}{l}\text { SN rate } \\
{\left[\mathrm{SN} \mathrm{yr}^{-1}\right]}\end{array}$ & $\begin{array}{l}\text { SFR } \\
{\left[M_{\odot} \mathrm{yr}^{-1}\right]}\end{array}$ \\
\hline $10^{4.5}$ & $3 \times 10^{-19}$ & 7.30 & 0.09 & 14.30 \\
& $5 \times 10^{-19}$ & 8.66 & 0.15 & 23.84 \\
$10^{5.0}$ & $2 \times 10^{-18}$ & 9.36 & 0.61 & 95.35 \\
& $9 \times 10^{-18}$ & 15.46 & 2.75 & 429.07 \\
$10^{5.5}$ & $9 \times 10^{-18}$ & 10.53 & 2.75 & 429.07 \\
& $2 \times 10^{-17}$ & 13.74 & 6.10 & 953.50 \\
\hline
\end{tabular}

By assuming a mechanical energy output of $10^{51}$ erg per SN, the $\mathrm{SN}$ rate can then be expressed as:

$\mathrm{SN}$ rate $=\frac{\mathrm{d} E_{\mathrm{SN}} / \mathrm{d} t}{3.17 \times 10^{43} \eta_{\text {trans }}} \mathrm{SN} \mathrm{y}^{-1}$

This yields $\mathrm{SN}$ rates ranging from $\sim 0.1 \mathrm{SN} \mathrm{yr}^{-1}$ for the $n=$ $10^{4.5} \mathrm{~cm}^{-3}$ models up to $\sim 6 \mathrm{SN} \mathrm{yr}^{-1}$ for the densest $(n=$ $10^{5.5} \mathrm{~cm}^{-3}$ ) clouds. A more convenient measure is to relate the $\mathrm{SN}$ rate with the star-formation rate (SFR), using the initial mass function (IMF) $\Phi(M)$ (Dahlén \& Fransson 1999):

$\frac{\mathrm{SN} \text { rate }}{\mathrm{SFR}}=k=\frac{\int_{8 M_{\odot}}^{50 M_{\odot}} \Phi(M) \mathrm{d} M}{\int_{0.1 M_{\odot}}^{125 M_{\odot}} M \Phi(M) \mathrm{d} M}$.

Using a Salpeter IMF gives a value of $k=0.0064$. To estimate a value for $f_{\mathrm{PDR}}$, we assume a region of size $\left(D_{\mathrm{SB}}\right) 100 \mathrm{pc}$ and an ambient density of $10^{3}-10^{4} \mathrm{~cm}^{-3}$, which corresponds to a total mass for the dense molecular gas in the SB region of $\sim 10^{6.5}-10^{7.5} M_{\odot}$. By assuming a clumping factor of 10 and using the same cloud size and densities as before, we obtain a $f_{\mathrm{PDR}}$ of about 15 PDRs $\mathrm{pc}^{-3}$. We note that only the product of $D_{\mathrm{SB}}$ and $f_{\mathrm{PDR}}$ is important for the SN rates and SFRs; a larger region with a lower PDR density will yield the same results, since we determine the total molecular gas mass that can be heated by SNe. The energy transfer efficiency $\eta_{\text {trans }}$ is assumed to be $10 \%$. A lower efficiency is not expected, since the amount of energy coming from the star-formation would otherwise become larger than the infra-red luminosities of the sources.

Table 2 lists the results of these calculations. It shows that the models that trace most of the data $\left(n=10^{4.5} \mathrm{~cm}^{-3}\right)$ require modest SFRs of only around $20 M_{\odot} \mathrm{yr}^{-1}$, whereas the models with higher densities require high SFRs. Such high SFRs are difficult to achieve and will disrupt the molecular region surrounding the star-forming region, which may correlate with the fact that there are no sources observed with line ratios corresponding to mechanically heated high-density PDRs.

Both YSO outflows and SNe can provide sufficient energy to heat the gas, but SNe are more likely to be the dominant process for the sources in our sample. First of all, SNe are able to inject the energy on large scales, redistributing the energy amongst the PDRs, while YSO outflows are only able to heat single clouds. This requires all stars and surrounding PDR clouds to be at the same (evolutionary) stage. Also, the time that an ensemble of stars exists in the phase where $\mathrm{SNe}$ are produced is far longer than the YSO stage, which lasts only $\sim 10^{5}-10^{6}$ years, implying that clouds heated by SN feedback are far more likely to be observed.

\section{Conclusions}

Although PDR, XDR or CR chemistry models can explain the general behavior of the $\mathrm{HCN}, \mathrm{HNC}$ and $\mathrm{HCO}^{+}$line ratios observed in nearby star-forming galaxies (Baan et al. 2008), they cannot explain the low $\mathrm{HNC} / \mathrm{HCN}$ line ratios observed in a large number of systems. The addition of heating by mechanical feedback provides a solution to this problem.

Our simulations in Figs. 1 and 2 show that the observed sources can be split into three main groups, based on their $\mathrm{HNC} / \mathrm{HCN}$ line ratios. First, there are a few sources that exhibit XDR chemistry $(\log (\mathrm{HNC} / \mathrm{HCN})>0)$. The other $(\mathrm{PDR})$ sources can be divided into two groups: a small group (composed mostly of the OH-MM and ULIRG systems) of sources that can be modeled with standard PDR chemistry and high $\left(n \geq 10^{5} \mathrm{~cm}^{-3}\right)$ densities and a larger group of sources with lower densities $\left(n \sim 10^{4.5} \mathrm{~cm}^{-3}\right)$ that are heated by mechanical feedback.

All evidence leads to the natural conclusion that the division between the PDR sources is a result of the evolution of the starformation cycle of a galaxy, during which the dominant heating process changes and density of the gas diminishes. The highdensity ( $n \geq 10^{5} \mathrm{~cm}^{-3}$ ) PDRs (with $\mathrm{HNC} / \mathrm{HCN}$ ratios around unity and weak $\mathrm{HCO}^{+}$lines) represent the early stage of starformation where the stars are forming in the densest clouds and the stellar UV radiation dominates the chemistry in those clouds. During this period most of the gas will be converted into stars and the increasing amount of UV radiation of the stars will gradually penetrate a larger volume of the (lower-density) gas. The resulting lower average density leads to an increase in $\mathrm{HCO}^{+}$ with respect to $\mathrm{HCN}$ and $\mathrm{HNC}$. After about $10 \mathrm{Myr}$, the first massive stars become $\mathrm{SNe}$ and their shocks begin to dominate the heating budget, leading to lower $\mathrm{HNC} / \mathrm{HCN}$ line ratios. These two effects alter the properties of the galaxies to those of a second group consisting of mechanical-feedback-dominated lowerdensity PDRs. Because of the short duty-cycle of the first stage compared to the second, most sources in our sample are observed to be in the later stage of their evolution.

It therefore appears that mechanical feedback plays a crucial role in the time dependent physical state of the dense starforming molecular gas in luminous infrared galaxies.

\section{References}

Aalto, S., Polatidis, A. G., Hüttemeister, S., \& Curran, S. J. 2002, A\&A, 381, 783

Baan, W. A., Henkel, C., Loenen, A. F., Baudry, A., \& Wiklind, T. 2008, A\&A, 477,747

Dahlén, T., \& Fransson, C. 1999, A\&A, 350, 349

Gao, Y., \& Solomon, P. M. 2004, ApJS, 152, 63

Graciá-Carpio, J., García-Burillo, S., Planesas, P., \& Colina, L. 2006, ApJ, 640, L135

Heckman, T. M., Armus, L., \& Miley, G. K. 1990, ApJS, 74, 833

Hollenbach, D. J., \& Tielens, A. G. G. M. 1999, Reviews of Modern Physics, 71, 173

Kohno, K., Muraoka, K., Hatsukade, B., et al. 2008, ArXiv e-prints, 805

Lepp, S., \& Dalgarno, A. 1996, A\&A, 306, L21

Maloney, P. R., Hollenbach, D. J., \& Tielens, A. G. G. M. 1996, ApJ, 466, 561

Meijerink, R., \& Spaans, M. 2005, A\&A, 436, 397

Meijerink, R., Spaans, M., \& Israel, F. P. 2006, ApJ, 650, L103

Meijerink, R., Spaans, M., \& Israel, F. P. 2007, A\&A, 461, 793

Mühle, S., Seaquist, E. R., \& Henkel, C. 2007, ApJ, 671, 1579

Nguyen-Q-Rieu, Jackson, J. M., Henkel, C., Truong, B., \& Mauersberger, R. 1992, ApJ, 399, 521

Ott, J., Weiss, A., Henkel, C., \& Walter, F. 2005, ApJ, 629, 767

Schilke, P., Walmsley, C. M., Pineau Des Forets, G., et al. 1992, A\&A, 256, 595

Solomon, P. M., Downes, D., \& Radford, S. J. E. 1992, ApJ, 387, L55

Talbi, D., Ellinger, Y., \& Herbst, E. 1996, A\&A, 314, 688 